\title{
Remarks on equality of two distributions under some partial orders
}

\author{
Chuancun Yin \\ School of Statistics, Qufu Normal University \\ Shandong 273165, China \\ e-mail: ccyin@mail.qfnu.edu.cn
}

October 18, 2018

\begin{abstract}
In this note we establish some appropriate conditions for stochastic equality of two random variables/vectors which are ordered with respect to convex ordering or with respect to supermodular ordering. Multivariate extensions of this result are also considered.
\end{abstract}

Keywords: Comonotonicity; Convex order; Distortion risk measure; Distortion function; Expected utility; Stop-loss order; Supermodular order

\section{INTRODUCTION}

Let $X$ and $Y$ be two random variables with distribution functions $F_{X}$ and $F_{Y}$ respectively. Let $\bar{F}_{X}$ and $\bar{F}_{Y}$ denote the corresponding survival functions. $X$ is said to precede $Y$ in the stop-loss order sense, notation $X \leq_{s l} Y$, if and only if $E\left[(X-d)_{+}\right] \leq E\left[(Y-d)_{+}\right],-\infty<$ $d<\infty ; X$ is said to precede $Y$ in the convex order sense, notation $X \leq_{c x} Y$, if and only if $X \leq_{s l} Y$ and in addition $E[X]=E[Y]$. Equivalently, $X \leq_{c x} Y$ if and only if $E f(X) \leq E f(Y)$ for every convex function $f$, provided that expectations $E f(X)$ and $E f(Y)$ exist. The stop-loss order can be characterized in terms of ordered TVaR's (see 
e.g. Dhaene et al. (2006)): $X \leq_{s l} Y \Leftrightarrow T \operatorname{TV} R_{p}[X] \leq T \operatorname{Va} R_{p}[Y]$ for all $p \in(0,1)$, where $T \operatorname{VaR}_{p}[X]=\frac{1}{1-p} \int_{p}^{1} F_{X}^{-1}(q) d q$ is the tail value-at -risk at level $p$, and $F_{X}^{-1}(q)=\inf \{x \in$ $\left.\mathbb{R} \mid F_{X}(x) \geq q\right\}$ with $\inf \emptyset=+\infty$, by convention. A random vector $Y=\left(Y_{1}, \cdots, Y_{n}\right)$ with marginal distributions $F_{Y_{i}}, i=1,2, \cdots, n$, is called commonotonic if

$$
Y \stackrel{d}{=}\left(F_{Y_{1}}^{-1}(U), F_{Y_{2}}^{-1}(U), \cdots, F_{Y_{n}}^{-1}(U)\right)
$$

where $\stackrel{d}{=}$ stands for "equality in distribution" and $U$ is a random variable that is uniformly distributed over the unit interval $(0,1)$. Consider a random vector $\left(Y_{1}, \cdots, Y_{n}\right)$ and its comonotonic counterpart $\left(Y_{1}^{c}, \cdots, Y_{n}^{c}\right)$. The sum of the components is denoted by $S$ and $S^{c}$ respectively. A nice result of Kaas et al. (2002) says that $S \leq_{c x} S^{c}$, and the converse remains valid by Theorem 4 in Cheung (2010); see Mao and $\mathrm{Hu}$ (2011) for a new proof. For more details about comonotonicity, stochastic orders and their applications, we refer the reader, e.g., to Joe (1997), Shaked and Shanthikumar (2007) and Denuit et al. (2005).

Cheung (2010) proved the following theorems giving sufficient conditions for stochastic equality of two random variables when these are known to be stochastically ordered.

Theorem 1.1. (Cheung (2010), Theorem 6) Let $Y_{1}$ and $Y_{2}$ be two integrable random variables and $u$ be any real-valued strictly convex function or strictly concave function which is twice continuously differentiable. Then

$$
Y_{1} \leq_{c x} Y_{2} \text { and } E\left[u\left(Y_{1}\right)\right]=E\left[u\left(Y_{2}\right)\right] \Rightarrow Y_{1} \stackrel{d}{=} Y_{2} .
$$

In particular,

$$
E[u(S)]=E\left[u\left(S^{c}\right)\right] \Leftrightarrow S \stackrel{d}{=} S^{c}
$$

Theorem 1.2. (Cheung (2010), Theorem 7) Let $Y_{1}$ and $Y_{2}$ be two integrable random variables, and $g$ be a strictly concave continuously differentiable distortion function with $g^{\prime}(0)<\infty$. Then

$$
Y_{1} \leq_{c x} Y_{2} \text { and } \rho_{g}\left[Y_{1}\right]=\rho_{g}\left[Y_{2}\right] \Rightarrow Y_{1} \stackrel{d}{=} Y_{2}
$$

In particular,

$$
\rho_{g}[S]=\rho_{g}\left[S^{c}\right] \Leftrightarrow S \stackrel{d}{=} S^{c}
$$

Cheung et al. (2015, Theorem 7) obtained the same result as in Theorem 1.1 under the following weaker conditions on $u$ : $u$ is a strictly concave (or strictly convex) function with absolutely continuous derivative $u^{\prime}$. Cheung et al. (2015, Theorem 8) obtained the same result as in Theorem 1.2 under the following more general conditions on the distortion 
function $g: g$ is continuously differentiable and strictly concave (or strictly convex). We remark that there is a very minor gap in the proof to Theorems 7 and 8 in Cheung et al. (2015). Our aim in this paper is to fill this gap and obtain more general sufficient conditions for stochastic equality of two random variables/vectors which are ordered with respect to the partial orders.

The rest of the paper is organized as follows. We review some basic definitions and notations such as convex and concave functions in Section 2. In Section 3 we characterize comonotonicity by distortion risk measures, and in Section 4 we characterize comonotonicity through expected utility. Finally, in Section 5 the multivariate extensions are considered.

\section{Some results for convex and concave functions}

Throughout the paper, we will use the notion $I$ to denote a nondegenerate interval of the real line. In this section, we present several concepts and results that will be used throughout the paper.

Definition 2.1 A function $f: I \rightarrow \mathbb{R}$ is called convex if

$$
f((1-\lambda) x+\lambda y) \leq(1-\lambda) f(x)+\lambda f(y)
$$

for all points $x$ and $y$ in $I$ and all $\lambda \in[0,1]$. It is called strictly convex if the inequality (2.1) holds strictly whenever $x$ and $y$ are distinct points and $\lambda \in[0,1]$. If $-f$ is convex (respectively, strictly convex) then we say that $f$ is concave (respectively, strictly concave).

Here are several elementary examples of convex functions of one variable:

- functions convex on the whole axis: $x^{2 r}, r$ being positive integer; $e^{t x}, t \neq 0 ;(x-$ $a)^{2}, a \in \mathbb{R}$.

- functions convex on the nonnegative ray: $x^{r}, r \geq 1 ;-x^{r}, 0 \leq r \leq 1 ; x \ln x$.

- functions convex on the positive ray: $x^{-r}, r>0 ;-\ln x$.

The following lemma is the result on the smoothness of convex functions, which can be found in Niculescu and Persson (2006, P. 21). 
Lemma 2.1. Let $f: I \rightarrow \mathbb{R}$ be a convex function. Then $f$ is continuous on the interior int(I) of $I$ and has finite left and right derivatives at each point of int(I). Moreover, $x<y$ in int(I) implies

$$
f_{-}^{\prime}(x) \leq f_{+}^{\prime}(x) \leq f_{-}^{\prime}(y) \leq f_{+}^{\prime}(y)
$$

Particularly, both $f_{-}^{\prime}$ and $f_{+}^{\prime}$ are nondecreasing on int $(I)$.

A convex function $f$ defined on some open interval $I$ is continuous on $I$ and Lipschitz continuous on any closed subinterval. $f$ admits left and right derivatives, and these are monotonically non-decreasing. As a consequence, $f$ is differentiable at all but at most countably many points. If $I$ is closed, then $f$ may fail to be continuous at the endpoints of $I$. For example, the function $f$ with domain $[0,1]$ defined by $f(0)=f(1)=1, f(x)=0$ for $0<x<1$ is convex; it is continuous on the open interval $(0,1)$, but not continuous at 0 and 1 .

Lemma 2.2. (The second derivative test) Suppose that $f: I \rightarrow \mathbb{R}$ is a twice differentiable function. Then:

(i) $f$ is convex if and only if $f^{\prime \prime} \geq 0$;

(ii) $f$ is strictly convex if and only if $f^{\prime \prime} \geq 0$ and the set of points where $f^{\prime \prime}$ vanishes does not include intervals of positive length.

A proof of this result can be found e.g., in Niculescu and Persson (2006).

Remark 2.1. An important result due to A. D. Alexandrov asserts that all convex functions are almost everywhere twice differentiable. See Theorem 3.11.2. in Niculescu and Persson (2006). Riesz-Nagy gave an example of real-valued function $\phi$ on $[0,1]$ such that $\phi(0)=0, \phi(1)=1, \phi$ is continuous and strictly increasing, and $\phi^{\prime}=0$ almost everywhere. See Hewitt and Stromberg (1965, Example 18.8, p. 278). Thus the function $u(x)=\int_{0}^{x} \phi(t) d t$ is strictly convex though $u^{\prime \prime}=0$ almost everywhere; see Niculescu and Persson (2006, P. 37).

\section{Convex order, expected utility and comonotonicity}

Definition 2.1 Let two measures $P$ and $Q$ be defined on the same space. $Q$ is called absolutely continuous with respect to $P$, written as $Q \ll P$, if $Q(A)=0$ whenever $P(A)=0$ for any measurable set $A$. $P$ and $Q$ are called equivalent if $Q \ll P$ and $P \ll Q$. 
Theorem 3.1. Let $Y_{1}$ and $Y_{2}$ be two integrable random variables on interval $I$, and $u: I \rightarrow \mathbb{R}$ be any convex function. Assume that $\lambda \ll \gamma$, where $\lambda$ is the Lebesgue measure on $\mathbb{R}$ and $\gamma$ is the positive Radon measure defined by

$$
\gamma(x, y]=u_{+}^{\prime}(y)-u_{+}^{\prime}(x) \text { for any } x<y
$$

where $u_{+}^{\prime}$ is the right-hand derivative of $u$. Then

$$
Y_{1} \leq_{c x} Y_{2} \text { and } E\left[u\left(Y_{1}\right)\right]=E\left[u\left(Y_{2}\right)\right] \Rightarrow Y_{1} \stackrel{d}{=} Y_{2} .
$$

In particular,

$$
E[u(S)]=E\left[u\left(S^{c}\right)\right] \Leftrightarrow S \stackrel{d}{=} S^{c} .
$$

By switching from $u$ to $-u$, yields that

Corollary 3.1. Let $Y_{1}$ and $Y_{2}$ be two integrable random variables on interval $I$, and $u: I \rightarrow \mathbb{R}$ be any concave function. Assume that $\lambda \ll \gamma$, where $\lambda$ is the Lebesgue measure on $\mathbb{R}$ and $\gamma$ is the positive Radon measure defined by

$$
\gamma(x, y]=u_{+}^{\prime}(x)-u_{+}^{\prime}(y) \text { for any } x<y
$$

where $u_{+}^{\prime}$ is the right-hand derivative of $u$. Then

$$
Y_{1} \leq_{c x} Y_{2} \text { and } E\left[u\left(Y_{1}\right)\right]=E\left[u\left(Y_{2}\right)\right] \Rightarrow Y_{1} \stackrel{d}{=} Y_{2} .
$$

In particular,

$$
E[u(S)]=E\left[u\left(S^{c}\right)\right] \Leftrightarrow S \stackrel{d}{=} S^{c} .
$$

Remark 3.1. If $u$ is convex and $u^{\prime \prime}>0$ almost everywhere or if $u$ is concave and $u^{\prime \prime}<0$ almost everywhere, or, more generally, $u$ is a any real-valued strictly convex or strictly concave function, then $\gamma$ is equivalent to $\lambda$. Thus Theorem 3.1 and Corollary 3.1 is generalization of Theorem 1.1 .

Remark 3.2. We remark that the proof to Theorem 7 in Cheung et al. (2015) has a gap if there is no further restrictions on $u$ (for example, $u^{\prime \prime}>0$ a.e. on I). In fact, the function $u$ in Remark 2.1 is an example of a strictly convex but $u^{\prime \prime}=0$ almost everywhere.

The proof of Theorem 3.1 requires the following lemma, which can be found in Föllmer and Schied (2004), see also Cheung (2010). 
Lemma 3.1. Suppose that $u$ is an increasing convex function with right-hand derivative $u_{+}^{\prime}$. There is a positive Radon measure $\gamma$ on $\mathbb{R}$ such that

$$
\gamma(x, y]=u_{+}^{\prime}(y)-u_{+}^{\prime}(x) \text { for any } x<y
$$

and

$$
\begin{aligned}
u(x)= & u(0)+u^{\prime}(0) x+\int_{(0, \infty)}(x-t)_{+} \gamma(d t) \\
& +\int_{(-\infty, 0]}(t-x)_{+} \gamma(d t), x \in \mathbb{R} .
\end{aligned}
$$

Proof of Theorem 3.1. We prove the theorem for the case where $u$ is increasing convex function only, the rest cases can be handled in a similar way as the proof to Theorem 6 in Cheung (2010). Notice that the convex order relation $Y_{1} \leq_{c x} Y_{2}$ implies that $E\left[Y_{1}\right]=E\left[Y_{2}\right]$. As in the step 1 of the proof to Theorem 6 in Cheung (2010), the condition $E\left[u\left(Y_{1}\right)\right]=E\left[u\left(Y_{2}\right)\right]$ imply that

$$
\int_{(0, \infty)}\left\{E\left(Y_{2}-t\right)_{+}-E\left(Y_{1}-t\right)_{+}\right\} \gamma(d t)+\int_{(-\infty, 0]}\left\{E\left(t-Y_{2}\right)_{+}-E\left(t-Y_{1}\right)_{+}\right\} \gamma(d t)=0 .
$$

Since $Y_{1} \leq_{c x} Y_{2}$, we have $E\left(Y_{2}-t\right)_{+}-E\left(Y_{1}-t\right)_{+} \geq 0$ and $E\left(t-Y_{2}\right)_{+}-E\left(t-Y_{1}\right)_{+} \geq 0$ for all $t$. It follows that $E\left(Y_{2}-t\right)_{+}=E\left(Y_{1}-t\right)_{+}$for $\gamma$-almost all $t>0$ and $E\left(t-Y_{2}\right)_{+}=E\left(t-Y_{1}\right)_{+}$ for $\gamma$-almost all $t \leq 0$, and hence $E\left(Y_{2}-t\right)_{+}=E\left(Y_{1}-t\right)_{+}$for $\lambda$-almost all $t>0$ and $E\left(t-Y_{2}\right)_{+}=E\left(t-Y_{1}\right)_{+}$for $\lambda$-almost all $t \leq 0$ since $\lambda \ll \gamma$. As the functions $E\left(Y_{i}-t\right)_{+}$ and $E\left(t-Y_{i}\right)_{+}$are continuous functions of $t$, we conclude that $Y_{1}$ and $Y_{2}$ have the same distribution.

\section{Convex order, distorted expectations and comono- tonicity}

A distortion function is a non-decreasing function $g:[0,1] \rightarrow[0,1]$ such that $g(0)=0$ and $g(1)=1$. The distorted expectation of the random variable $X$ associated with distortion function $g$, notation $\rho_{g}[X]$, is defined as

$$
\rho_{g}[X]=\int_{0}^{+\infty} g\left(\bar{F}_{X}(x)\right) d x+\int_{-\infty}^{0}\left[g\left(\bar{F}_{X}(x)\right)-1\right] d x,
$$


provided at least one of the to integrals above is finite. If $X$ a non-negative random variable, then $\rho_{g}$ reduces to

$$
\rho_{g}[X]=\int_{0}^{+\infty} g\left(\bar{F}_{X}(x)\right) d x .
$$

In view of Dhaene et al. (2012, Theorems 4 and 6) we know that, when the distortion function $g$ is right continuous on $[0,1)$, then $\rho_{g}[X]$ may be rewritten as

$$
\rho_{g}[X]=\int_{[0,1]} \operatorname{VaR} R_{1-q}^{+}[X] d g(q),
$$

where $\operatorname{VaR}^{+} p[X]=\sup \left\{x \mid F_{X}(x) \leq p\right\}$, and when the distortion function $g$ is left continuous on $(0,1]$, then $\rho_{g}[X]$ may be rewritten as

$$
\rho_{g}[X]=\int_{[0,1]} \operatorname{VaR}_{1-q}[X] d g(q)=\int_{[0,1]} \operatorname{VaR}_{q}[X] d \bar{g}(q),
$$

where $\operatorname{VaR}_{p}[X]=\inf \left\{x \mid F_{X}(x) \geq p\right\}$ and $\bar{g}(q):=1-g(1-q)$ is the dual distortion of $g$. Obviously, $\overline{\bar{g}}=g, g$ is left continuous if and only if $\bar{g}$ is right continuous; $g$ is concave if and only if $\bar{g}$ is convex.

Theorem 4.1. Let $Y_{1}$ and $Y_{2}$ be two integrable random variables, and $g$ be a concave distortion function. Assume that $\lambda \ll \nu$, where $\lambda$ is the Lebesgue measure on $\mathbb{R}$ and $\nu$ is the Radon measure defined by $\nu([0, q])=g_{+}^{\prime}(1-q)$. Then we have that

$$
Y_{1} \leq_{c x} Y_{2} \text { and } \rho_{g}\left[Y_{1}\right]=\rho_{g}\left[Y_{2}\right] \Rightarrow Y_{1} \stackrel{d}{=} Y_{2}
$$

In particular,

$$
\rho_{g}[S]=\rho_{g}\left[S^{c}\right] \Leftrightarrow S \stackrel{d}{=} S^{c}
$$

Proof The distortion measure with concave distortion function $g$ can be expressed by the weighted TVaR. In fact, note that $\phi(q)=g_{+}^{\prime}(1-q)$ is monotone increasing, so $\nu([0, q])=\phi(q)$ is positive measure. We have

$$
\begin{aligned}
\rho_{g}[X] & =-\int_{0}^{1} \operatorname{VaR}_{w}[X] d g(1-w) \\
& =\int_{0}^{1} \operatorname{VaR}_{w}[X] g_{+}^{\prime}(1-w) d w \\
& =\int_{0}^{1} \operatorname{VaR}_{w}[X] \phi(w) d w \\
& =\nu([0,1]) E X+\int_{0}^{1} T \operatorname{Ta} R_{w}[X](1-w) d \nu(w) \\
& =\nu([0,1]) E X+\int_{0}^{1} \operatorname{TVaR}_{w}[X] d \mu(w),
\end{aligned}
$$


where

$$
d \mu(w)=(1-w) d \nu(w)
$$

It can be shown that $\mu$ is a probability measure. In fact,

$$
\begin{aligned}
\int_{0}^{1} d \mu(w) & =\int_{0}^{1} \nu([0, w]) d w \\
& =\int_{0}^{1} \phi(w) d w=\int_{0}^{1} g_{+}^{\prime}(w) d w=1 .
\end{aligned}
$$

The convex order $Y_{1} \leq_{c x} Y_{2}$ implies that $E Y_{1}=E Y_{2}$ and $T V a R_{p}\left[Y_{1}\right] \leq T V a R_{p}\left[Y_{2}\right]$, for all $p \in(0,1)$. As in Cheung et al. (2015) we have

$$
\begin{aligned}
0 & =\rho_{g}\left[Y_{1}\right]-\rho_{g}\left[Y_{1}\right] \\
& =\int_{0}^{1} T V a R_{w}\left[Y_{1}\right](1-w) d \nu(w)-\int_{0}^{1} T \operatorname{Va} R_{w}\left[Y_{2}\right](1-w) d \nu(w)
\end{aligned}
$$

We conclude that $T \operatorname{Va} R_{p}\left[Y_{1}\right]=T V a R_{p}\left[Y_{2}\right]$, for $\nu$-almost all $p \in(0,1)$, and hence $T \operatorname{VaR}_{p}\left[Y_{1}\right]=T \operatorname{VaR}_{p}\left[Y_{2}\right]$, for $\lambda$-almost all $p \in(0,1)$ since $\lambda \ll \nu$. Furthermore, as the function $T V a R_{p}\left[Y_{1}\right]-T V a R_{p}\left[Y_{2}\right]$ is a continuous function of $p$, we have $T \operatorname{Va} R_{p}\left[Y_{1}\right]=$ $T \operatorname{TVaR} R_{p}\left[Y_{2}\right]$, for all $p \in(0,1)$, which is equivalent with $E\left(Y_{2}-t\right)_{+}=E\left(Y_{1}-t\right)_{+}$for all $t \in \mathbb{R}$. Thus $Y_{1} \stackrel{d}{=} Y_{2}$.

Corollary 4.1. Let $Y_{1}$ and $Y_{2}$ be two integrable random variables, and $g$ be a strictly convex distortion function. Assume that $\lambda \ll \nu$, where $\lambda$ is the Lebesgue measure on $\mathbb{R}$ and $\nu$ is the Radon measure defined by $\nu([0, q])=-g_{+}^{\prime}(1-q)$. Then we have that

$$
Y_{1} \leq_{c x} Y_{2} \text { and } \rho_{g}\left[Y_{1}\right]=\rho_{g}\left[Y_{2}\right] \Rightarrow Y_{1} \stackrel{d}{=} Y_{2}
$$

In particular,

$$
\rho_{g}[S]=\rho_{g}\left[S^{c}\right] \Leftrightarrow S \stackrel{d}{=} S^{c}
$$

Remark 4.1. If $g$ is a any real-valued strictly convex or strictly concave function, then $\nu$ is equivalent to $\lambda$. Thus Theorem 4.1 is generalization of Theorem 1.2.

Remark 4.2. Theorem 8 in Cheung et al. (2015) obtained the above results under conditions that $g$ is a strictly concave (or strictly convex) distortion function with absolutely continuous derivative $g^{\prime}$. We remark that, as in Remark 3.2, the proof to Theorem 8 in Cheung et al. (2015) has a minor gap if there is no further restrictions on $g$ (for example, $g^{\prime \prime}>0$ a.e. on $\left.[0,1]\right)$. In fact, the function $g(x)=\frac{\int_{0}^{x} \phi(t) d t}{\int_{0}^{1} \phi(t) d t}$ is strictly increasing distortion function, but $u^{\prime \prime}=0$ almost everywhere, where the function $\phi$ is defined in Remark 2.1. 
Remark 4.3. As remarked in Cheung (2010), the condition $Y_{1} \leq_{c x} Y_{2}$ in Theorem 4.1 can be slightly relaxed to $Y_{1} \leq_{s l} Y_{2}$. In fact, as is well known $Y_{1} \leq_{s l} Y_{2}$ implies that $E Y_{1} \leq E Y_{2}$. Moreover, $Y_{1} \leq_{s l} Y_{2} \Leftrightarrow T V a R_{p}\left[Y_{1}\right] \leq T V a R_{p}\left[Y_{2}\right]$ for all $p \in(0,1)$ (see, e.g. Dhaene et al. (2006), Theorem 3.2). By using (4.1) we have

$$
\begin{aligned}
0 & =\rho_{g}\left[Y_{1}\right]-\rho_{g}\left[Y_{1}\right] \\
& =\nu([0,1])\left(E Y_{1}-E Y_{2}\right)+\int_{0}^{1}\left(T V a R_{w}\left[Y_{1}\right]-T V a R_{w}\left[Y_{2}\right]\right) d \mu(w),
\end{aligned}
$$

which implies $E Y_{1}=E Y_{2}$ and $\int_{0}^{1}\left(T V a R_{w}\left[Y_{1}\right]-T V a R_{w}\left[Y_{2}\right]\right) d \mu(w)=0$. Thus $Y_{1} \stackrel{d}{=} Y_{2}$.

\section{Multivariate extensions}

As in Cheung et al. (2015) we use the notions $\underline{X}$ and $\underline{Y}$ to denote the $n$-vectors $\left(X_{1}, X_{2}, \cdots, X_{n}\right)$ and $\left(Y_{1}, Y_{2}, \cdots, Y_{n}\right)$, respectively. The sums of their components are denoted by $S_{X}$ and $S_{Y}$, respectively:

$$
S_{X}=X_{1}+\cdots+X_{n} \text {, and } S_{Y}=Y_{1}+\cdots+Y_{n}
$$

Definition 5.1 A function $f: \mathbb{R}^{n} \rightarrow \mathbb{R}$ is said to be supermodular if for any $\underline{X}, \underline{Y} \in \mathbb{R}^{n}$ it satisfies

$$
f(\underline{X})+f(\underline{Y}) \leq f(\underline{X} \wedge \underline{Y})+f(\underline{X} \vee \underline{Y}),
$$

where the operators $\vee$ and $\wedge$ denote coordinatewise minimum and maximum, respectively.

$\underline{X}$ is said to be smaller in the supermodular order that $\underline{Y}$, notation $\underline{X} \leq_{S M} \underline{Y}$, if $E f(\underline{X}) \leq$ $E f(\underline{Y})$ holds for all supermodular functions $f: \mathbb{R}^{n} \rightarrow \mathbb{R}$ for which the expectations exist.

Parallel to the Theorems 13 and 14 in Cheung et al. (2015), we have the following two theorems under weaker conditions on $u$ and $g$.

Theorem 5.1. Consider the $n$-vectors $\underline{X}$ and $\underline{Y}$ with respective sums $S_{X}$ and $S_{Y}$ which are assumed to have finite expectations. Furthermore, consider the interval I with $P\left(S_{Y} \in\right.$ $I)=1$, and $u: I \rightarrow \mathbb{R}$ be any concave function. Assume that $\lambda \ll \gamma$, where $\lambda$ is the Lebesgue measure on $\mathbb{R}$ and $\gamma$ is the positive Radon measure defined by

$$
\gamma(x, y]=u_{+}^{\prime}(x)-u_{+}^{\prime}(y) \text { for any } x<y,
$$


where $u_{+}^{\prime}$ is the right-hand derivative of $u$. Finally, suppose that either

$$
E\left[\max \left(S_{Y}, 0\right)\right]^{n-1}<\infty \text { or } E\left[-\min \left(S_{Y}, 0\right)\right]^{n-1}<\infty
$$

Then we have that

$$
\underline{X} \leq_{S M} \underline{Y} \text { and } E\left[u\left(S_{X}\right)\right]=E\left[u\left(S_{Y}\right)\right] \Rightarrow \underline{X} \stackrel{d}{=} \underline{Y} .
$$

Theorem 5.2. Consider the $n$-vectors $\underline{X}$ and $\underline{Y}$ with respective sums $S_{X}$ and $S_{Y}$ which are assumed to have finite expectations. Furthermore, let $g$ be a concave distortion function. Assume that $\lambda \ll \nu$, where $\lambda$ is the Lebesgue measure on $\mathbb{R}$ and $\nu$ is the Radon measure defined by $\nu([0, q])=g^{\prime}(1-q)$. Finally, suppose that either

$$
E\left[\max \left(S_{Y}, 0\right)\right]^{n-1}<\infty \text { or } E\left[-\min \left(S_{Y}, 0\right)\right]^{n-1}<\infty .
$$

Then we have that

$$
\underline{X} \leq_{S M} \underline{Y} \text { and } \rho_{g}\left[S_{X}\right]=\rho_{g}\left[S_{Y}\right] \Rightarrow \underline{X} \stackrel{d}{=} \underline{Y}
$$

Remark 5.1. By switching from $u$ to $-u$ in Theorems 5.1 and 5.2 we can obtain the versions for convex functions $u$ and $g$.

Acknowledgements. The research was supported by the National Natural Science Foundation of China (No. 11171179), the Research Fund for the Doctoral Program of Higher Education of China (No. 20133705110002) and the Program for Scientific Research Innovation Team in Colleges and Universities of Shandong Province.

\section{References}

[1] Cheung, K. C. (2010). Characterizing a comonotonic random vector by the distribution of the sum of its components. Insurance: Mathematics and Economics 47(2), 130-136.

[2] Cheung, K. C., Dhaene, J., Kukush, A., Linders, D. (2015). Ordered random vectors and equality in distribution. Scandinavian Actuarial Journal, 2015(3), 221-244.

[3] Denuit, M., Dhaene, J., Goovaerts, M.J., Kaas, R. (2005). Actuarial Theory for Dependent Risks: Measures, Orders and Models. John Wiley \& Sons, Inc. 
[4] Dhaene, J., Denuit, M., Goovaerts, M., Kaas, R., Vyncke, D. (2002). The concept of comonotonicity in actuarial science and finance: theory. Insurance: Mathematics and Economics 31(1), 3-33.

[5] Dhaene, J., Kukush, A., Linders, D., Tang, Q. (2012). Remarks on quantiles and distortion risk measures. European Actuarial Journal 2, 319-328.

[6] Dhaene, J., Vanduffel, S., Goovaerts, M.J., Kaas, R., Tang, Q., Vyncke, D. (2006). Risk measures and comonotonicity: a review. Stochastic Models 22, 573-606.

[7] Fölmer, H., Schied, A. (2004). Stochastic Finance. Walter de Gruyter \& Co., Berlin.

[8] Hewitt, E., Stromberg, K. (1965). Real and Abstract Analysis. Springer- Verlag, Berlin.

[9] Joe, H. (1997). Multivariate models and dependence concepts. Chapman \& Hall, London.

[10] Mao, T., Hu, T. (2011). A new proof of Cheung's characterization of comonotonicity. Insurance: Mathematics and Economics 48(2), 214-216.

[11] Niculescu, C. P., Persson, L. E. (2006). Convex Functions and Their Applications: A Contemporary Approach. Springer, New York.

[12] Shanthikumar, J. G., Shaked, M. (2007). Stochastic orders. Springer, New York. 